\title{
Modified Equation of Motion Approach for Ferromagnetic Systems
}

\begin{abstract}
G. Górski*, J. Mizia And K. KuCAB
Faculty of Mathematics and Natural Sciences, University of Rzeszów, S. Pigonia 1, 35-310 Rzeszów, Poland

The key problem in using the dynamical mean-field theory is finding the appropriate solution to the single impurity Anderson model. We use the modified equation of motion method based on differentiation over two time variables. In such approach we obtain correct description of the Kondo effect not only for systems symmetrical with respect to the half-filled point but also for the systems without symmetry in a half filled point and for broad range of electron concentrations like e.g. the ferromagnetic systems. For the reason of these advantages we investigate in this report dependence of the system magnetic moment on carrier concentration using our modified equation of motion method. We also analyze influence of asymmetric densities of states on ferromagnetic alignment. Our results are compared with dynamical mean-field theory-quantum Monte Carlo calculations and with Hubbard I and III approximations.
\end{abstract}

DOI: 10.12693/APhysPolA.127.207

PACS: 71.10.Fd, 75.10.Lp, 75.30.Kz

\section{Introduction}

The Hubbard model was introduced to describe correlation effects in transition metals and particularly metallic ferromagnetism. Despite its simplicity there is no exact solution in 3D except for some specific cases (e.g. the Nagaoka solution). One of the well justified solving methods is the dynamical mean-field theory. The key problem in this method is to solve properly the impurity problem. For the ferromagnetic systems this problem was solved by the numerical methods (e.g. quantum Monte Carlo (QMC) [1]) and also analytically (e.g. iterative perturbation theory (IPT) [2] and modified iterative perturbation theory (MPT) [3]). All these methods have some limitations. The modified iterative perturbation theory relies on fitting the self-energy calculated in the second order perturbation theory to the atomic limit at arbitrary band-fillings.

In this paper we present the DMFT solutions for ferromagnetic system with single-impurity Anderson model (SIAM) solver obtained from modified equation of motion. Our results are similar to those given by the MPT method, but the expression for the self energy is derived from equations of motion. It is not given by interpolation scheme which is used in the MPT method. We calculate dependence of the Curie temperature on electron concentration and we investigate influence of DOS asymmetry on the range of existence of ferromagnetism. The results are compared with those obtained by Ulmke [1] in the QMC method.

\section{The model}

The Hamiltonian for the single band Hubbard model in a pure itinerant system can be written in the form

\footnotetext{
*corresponding author; e-mail: ggorski@univ.rzeszow.pl
}

$$
\begin{aligned}
H & =-t \sum_{<i j>\sigma}\left(c_{i \sigma}^{+} c_{j \sigma}+H . c .\right)-\sum_{i \sigma}(\mu+\sigma B) \hat{n}_{i \sigma} \\
& +\frac{U}{2} \sum_{i \sigma} \hat{n}_{i \sigma} \hat{n}_{i-\sigma},
\end{aligned}
$$

where $c_{i \sigma}^{+}\left(c_{i \sigma}\right)$ is the operator creating (destroying) an electron with spin $\sigma$ on the $i$-th lattice site, $t$ is the nearest-neighbors hopping integral, $\mu$ is the chemical potential and $B$ is a homogeneous external magnetic field. The potential part of the Hamiltonian (1) contains the on-site Coulomb repulsion $U=(i, i|1 / r| i, i)$. The solution to the Hubbard model is the on-site Green function $G_{i i \sigma}(\varepsilon)=\left\langle\left\langle c_{i \sigma} ; c_{i \sigma}^{+}\right\rangle\right\rangle_{\varepsilon}$ given by

$$
G_{i i \sigma}(\varepsilon)=\int_{-\infty}^{\infty} \frac{\rho_{0}\left(\varepsilon^{\prime}\right) \mathrm{d} \varepsilon^{\prime}}{\varepsilon+\mu_{\sigma}-\varepsilon^{\prime}-\Sigma_{\sigma}(\varepsilon)},
$$

where $\Sigma_{\sigma}(\varepsilon)$ is the local self-energy, $\mu_{\sigma}=\mu+\sigma B$ and $\rho_{0}\left(\varepsilon^{\prime}\right)$ denotes the unperturbed density of states. Now the problem is reduced to calculating the quantity $\Sigma_{\sigma}(\varepsilon)$. At present the widely used method of calculating the local self-energy is the dynamical mean-field theory. The DMFT is based on replacing the Hubbard model (1) by SIAM. The solution of SIAM problem is the impurity Green function $G_{d \sigma}(\varepsilon)$ given by

$$
G_{d \sigma}(\varepsilon)=\frac{1}{\varepsilon+\mu_{\sigma}-\Delta_{\sigma}(\varepsilon)-\Sigma_{d \sigma}},
$$

where $\Delta_{\sigma}(\varepsilon)$ is the hybridization function. Next basic assumption of the DMFT is the condition that the Green function of SIAM problem has to be the same as that of the extended medium: $G_{d \sigma}(\varepsilon)=G_{i i \sigma}(\varepsilon)$. This allows to write the hybridization function in a form

$$
\Delta_{\sigma}(\varepsilon)=\varepsilon+\mu_{\sigma}-\Sigma_{d \sigma}-\frac{1}{G_{i i \sigma}(\varepsilon)} .
$$

For calculating the self-energy we will use the equation of motion approach. This method was widely used in analysis of the SIAM problem, but until now the differentiation was performed over one time variable only what 
led to the following equation:

$$
\varepsilon\langle\langle A ; B\rangle\rangle_{\varepsilon}=\left\langle[A, B]_{+}\right\rangle+\left\langle\left\langle[A, H]_{-} ; B\right\rangle\right\rangle_{\varepsilon} .
$$

In this report we will differentiate also over the second time variable $\left(t^{\prime}\right)$, which gives the following form of EOM [4]:

$$
-\varepsilon\langle\langle A ; B\rangle\rangle_{\varepsilon}=-\left\langle[A, B]_{+}\right\rangle+\left\langle\left\langle A ;[B, H]_{-}\right\rangle\right\rangle_{\varepsilon} .
$$

Using both these equations we arrive at the self-energy in the form (for details see $[5,6]$ ):

$$
\Sigma_{d \sigma}(\varepsilon)=U n_{d-\sigma}+\frac{U^{2} \Gamma_{d \sigma}(\varepsilon)}{1+\alpha_{\sigma} U^{2} \Gamma_{d \sigma}(\varepsilon)},
$$

where

$$
\begin{aligned}
& \Gamma_{\sigma}(\varepsilon, T)=\iiint \frac{S_{d-\sigma}^{\mathrm{HF}}(x) S_{d-\sigma}^{\mathrm{HF}}(y) S_{d \sigma}^{\mathrm{HF}}(z)}{\varepsilon+x-y-z+i 0^{+}} \\
& \quad \times[f(x) f(-y) f(-z)+f(-x) f(y) f(z)] \mathrm{d} x \mathrm{~d} y \mathrm{~d} z,
\end{aligned}
$$
and

$$
\alpha_{\sigma}=
$$

$$
\frac{\mu_{\sigma}^{\mathrm{HF}}-\mu_{\sigma}+U / 2\left(1-2 n_{d-\sigma}\right)}{\left(\mu_{\sigma}^{\mathrm{HF}}-\mu_{\sigma}\right)\left(\mu_{\sigma}^{\mathrm{HF}}-\mu_{\sigma}+U-2 U n_{d-\sigma}\right)-U^{2} n_{d-\sigma}\left(1-n_{d-\sigma}\right)} .
$$

In Eq. (8) we use the $\mathrm{H}-\mathrm{F}$ spectral density $S_{d \sigma}^{\mathrm{HF}}(\varepsilon)=$ $-\frac{1}{\pi} \operatorname{Im} G_{d \sigma}^{\mathrm{HF}}(\varepsilon)$, with

$$
G_{d \sigma}^{\mathrm{HF}}(\varepsilon)=\frac{1}{\varepsilon+\mu_{\sigma}^{\mathrm{HF}}-\Delta_{\sigma}-U n_{-\sigma}} .
$$

\section{Numerical results and discussion}

In our numerical analysis we will concentrate on correlation between DOS asymmetry and the range of existence of ferromagnetism. As the non-interacting DOS we will use the following formula [7]:

$$
\rho_{0}(\varepsilon)=\frac{1+\sqrt{1-a_{1}^{2}}}{\pi D} \frac{\sqrt{D^{2}-\varepsilon^{2}}}{D+a_{1} \varepsilon},
$$

with the asymmetry parameter $a_{1}$ varying continuously from $a_{1}=0$, which is the case corresponding to a symmetric semi-elliptic band (or the Bethe lattice) to $a_{1} \approx 1$ corresponding to a fcc lattice. Parameter $D$ is a half bandwidth of the symmetric semi-elliptic band and will be assumed as the unit of energy $(D=1)$.

In Fig. 1 we present dependence of the Curie temperature on carrier concentration for moderately asymmetric DOS with $a_{1}=0.5$. The Curie temperature is obtained based on the temperature dependence of the spontaneous magnetization $m=n_{\uparrow}-n_{\downarrow}$ in the limit of an infinitesimally small applied field $B$. For the Curie temperature we use the condition $m\left(T_{\mathrm{C}}\right) \rightarrow 0$. We assume that the Coulomb interaction $U=4$. The $U / D$ ratio is the same as used by Wahle et al. [7] $(U / D \approx 2-4)$ and is similar to the one used by Ulmke [1]. Similar ratios $U / D$ are observed also in the $3 d$ transition metals.

Increase of the Coulomb interaction and particularly increase of asymmetry parameter enhance the ferromagnetism. At $a_{1}=0.7, U=4$ and $T=0.01$ we obtain the ferromagnetic ordering at electron concentration $n<$ 0.74 , and for $a_{1}=0.97$ already at $n<0.83$. The strong DOS asymmetry decreases the "re-entering" effect of ferromagnetism, which is the poor feature of flat DOS.

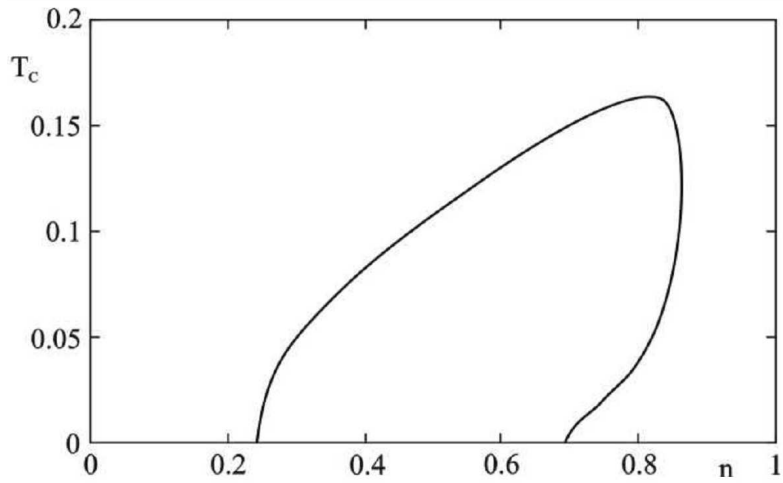

Fig. 1. Curie temperature as a function of carrier concentration $n$ for Coulomb interaction $U=4$ and DOS asymmetry parameter $a_{1}=0.5$.

For comparison with the QMC [1] and MPT [8] methods we present in Fig. 2 the dependence of the Curie temperature on carrier concentration for the $d=\infty$ fcc lattice with DOS given in [1]. The results presented by Ulmke [1] show smaller values of the Curie temperature but the smaller range of existence of ferromagnetic ordering as compared to our predictions. Our results are close to those obtained by the IPT method. In the classic version of this method [2] the values of the Coulomb repulsion critical for transition to ferromagnetic phase are higher than in our approach and in QMC method. Nolting et al. [8], who have used the MPT method [8], obtained magnetism at smaller values of $U$ by including the band-shift correlation function. Their results points towards ferromagnetism in the broader range of concentrations and with smaller Curie temperatures than ours.

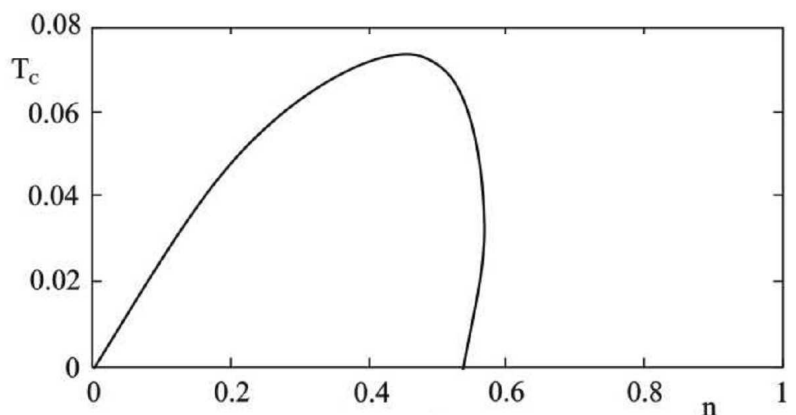

Fig. 2. Curie temperature as a function of carrier concentration $n$ for the $d=\infty$ fcc lattice [1] and Coulomb interaction $U=2$.

The Hubbard I and Hubbard III approximations were based on the EOM method in the form given by Eq. (5). The ferromagnetic state was obtained by including the inter-site correlations which led to the bandwidth and band-shift effects (see [8,9]). The present approach does not need the inter-site correlations to acquire ferromagnetism, but use of these correlations in the MPT method 
(see $[3,8])$ enhances ferromagnetic state in comparison to the classic IPT results.

In summary, using alternative EOM approach we obtained DOS with the three peaks structure composed of the quasiparticle resonance peak at the Fermi energy (Kondo peak). This method was used to describe metallic ferromagnetism in the single band Hubbard model within the dynamical mean-field theory. It was shown that the increase of DOS asymmetry strongly increases the concentration range of ferromagnetism and the values of the Curie temperature. Our results resemble those predicted by other methods (e.g. MPT, QMC) but as opposed to the MPT method our self-energy comes directly from the equation of motion for Green functions and not from the interpolation scheme.

The EOM approach used here to solve the SIAM problem can be easily applied to describe ferromagnetism in $R_{2} \mathrm{Fe}_{14} \mathrm{~B}(R=\mathrm{Nd}, \mathrm{Y})[10]$ compounds which are relatively novel permanent magnet materials. In these compounds there is a strong interaction between localized $R(4 f)$ states and itinerant $\mathrm{Fe}(3 d)$ states. After taking into account the hybridization between $R(4 f)$ and $\mathrm{Fe}(3 d)$ states we obtain the periodic Anderson model (PAM) which can be treated by EOM approach developed here. Further work in this direction is in progress.

\section{Acknowledgments}

This work was done due to partial support from Centre for Innovation and Transfer of Natural Sciences and Engineering Knowledge of Rzeszów University.

\section{References}

[1] M. Ulmke, Eur. Phys. J. B 1, 301 (1998).

[2] H. Kajueter, G. Kotliar, Phys. Rev. Lett. 77, 131 (1996).

[3] M. Potthoff, T. Wegner, W. Nolting, Phys. Rev. B 55, 16132 (1997).

[4] A.L. Kuzemsky, Riv. Nuovo Cimento 25, 1 (2002).

[5] G. Górski, J. Mizia, Physica B 427, 42 (2013).

[6] G. Górski, J. Mizia, K. Kucab, arXiv:1404.4439.

[7] J. Wahle, N. Blümer, J. Schlipf, K. Held, D. Vollhardt, Phys. Rev. B 58, 12749 (1998).

[8] W. Nolting, M. Potthoff, T. Herrmann, T. Wegner, in: Band-Ferromagnetism, Eds. K. Baberschke, M. Donath, W. Nolting, Springer, Berlin 2001, p. 208.

[9] G. Górski, J. Mizia, Phys. Rev. B 83, 064410 (2011).

[10] J.F. Herbst, J.J. Croat, F.E. Pinkerton, W.B. Yelon, Phys. Rev. B 29, 4176 (1984). 